\title{
La utilización de plásticos y su impacto ambiental en las Islas Galápagos.
}

The use of plastics and their environmental impact in the Galapagos Islands.

\author{
Dr. Tatiana Carrasco R. ${ }^{1}$
}

\section{Resumen.}

Las islas Galápagos son archipiélagos que se encuentran situadas a 1.050 kilómetros de la costa del Ecuador, su capital Puerto Baquerizo Moreno y turísticamente son conocidas como las islas encantadas, se estima que la formación de la primera Isla tuvo lugar hace más de 5 millones de años, como resultado de la actividad tectónica, los animales de las Islas Galápagos son generalmente clasificados como especies nativas, endémicas o introducidas, las especies endémicas son animales que solo se encuentran en las Islas Galápagos y en ninguna otra parte del mundo en un habitad natural, un buen ejemplo de esto sería el cormorán no volador, los animales nativos son animales que se encuentran de forma natural en las Islas Galápagos, pero también se encuentran de forma natural en otros lugares del mundo, como el Piquero de Nazca, en cambio las especies introducidas son especies en las Islas Galápagos, que no se encuentran en su habitad natural, como las ratas, perros y chivos o cabras. Como todos sabemos las Islas Galápagos su fauna y flora son únicas en el mundo, pero poco a poco estamos terminando con su ecosistema y biodiversidad ya que existe una gran cantidad de basura plástica en el océano y está llegando a nuestro archipiélago en gran cantidad, es un problema muy grande en las islas ya que esto perjudica y mata a las especies endémicas y a todo su ecosistema. Los plásticos que diariamente utilizamos y desechamos toman mucho tiempo en degradarse esto ocasiona que animales confundan la comida con este tipo de basura platica tanto así que les causa la muerte o por el simple hecho de cogerlos como objeto de juego se enredan y quedan atrapados se lastiman y mueren, ocasionando de esta manera un gran Impacto Ambiental.

Palabras claves: Impacto ambiental, Plástico, Galápagos.

\section{Abstract.}

\footnotetext{
${ }^{1}$ Visionario digital, Ambato,Ecuador,tatianacarrasco@cienciadigital.org
} 
The Galapagos Islands are archipelagos that are located 1,050 kilometers from the coast of Ecuador, its capital Puerto Baquerizo Moreno and tourism are known as the enchanted islands, it is estimated that the formation of the first island took place more than 5 million years ago As a result of the tectonic activity, the animals of the Galapagos Islands are generally classified as native, endemic or introduced species, the endemic species are animals that are only found in the Galapagos Islands and nowhere else in the world in a natural habitat, A good example of this would be the flightless cormorant, the native animals are animals that are naturally found in the Galapagos Islands, but they are also found naturally in other parts of the world, such as the Nazca Pike, instead the species introduced are species in the Galapagos Islands, which are not found in their natural habitat, such as rats, dogs and goats or Goats As we all know the Galapagos Islands, its fauna and flora are unique in the world, but little by little we are ending up with its ecosystem and biodiversity as there is a large amount of plastic garbage in the ocean and it is reaching our archipelago in great quantity. a very big problem in the islands since this harms and kills the endemic species and their entire ecosystem. The plastics that we use daily and discard take a long time to degrade this causes animals to confuse the food with this type of garbage talk so much that it causes their death or by the simple fact of catching them as a game object they become entangled and get trapped they get hurt and they die, causing in this way a great Environmental Impact.

Keywords: Environmental impact, Plastic, Galapagos.

\section{Introducción}

Según: (Allinson R. 2014., CALLEJA D. 2018., FERNÁNDEZ J. 2014., Iván V. 2016., Loustaunau M. 2014 \& Padova U. 2014). Aseguran que, el impacto ambiental es cualquier modificación del medio ambiente, sea adversa o beneficiosa, como resultado una alteración o efecto producido por el hombre en su entorno natural, de esta forma el impacto negativo represente una ruptura en el equilibrio ecológico, causando graves daños y perjuicios en el medio ambiente, así como en la salud de las personas y demás seres vivíos. La generación de residuos asociados a los aparatos eléctricos y electrónicos que aumenta todos los años a nivel mundial, esta basura electrónica es la causante de un impacto ambiental considerable que, en algunos casos también puede llegar a afectar la salud de las personas que la manipulan.

Imagen N. 1: Barco Jessica- Derrame de Combustible- Impacto Ambiental.

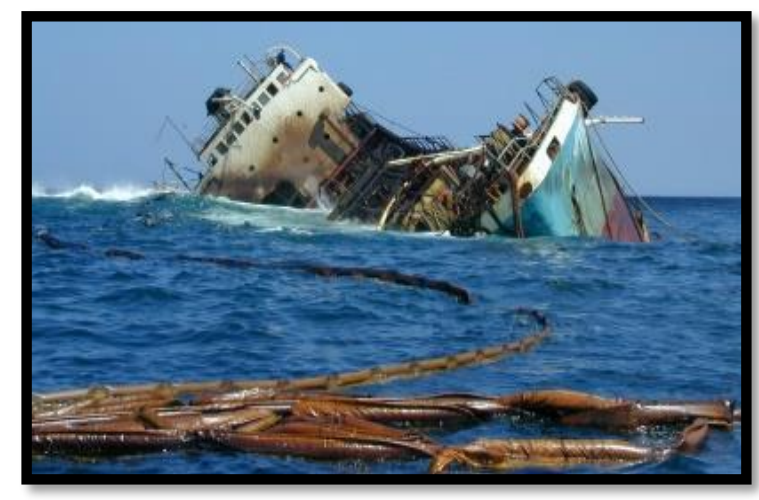

Fuente: https://waste.ideal.es/galapagos.htm 
Los cambios ocurridos actualmente en nuestro medio es por el impacto ambiental que es el daño al planeta tierra, uno de los problemas más grandes en el mundo que está afectando no solo al medio ambiente sino también a las personas que habitamos en ella y este, es generado por el hombre ya que no tomamos conciencia del daño que le hacemos al planeta tierra con la contaminación, la sobrepoblación la deforestación los derrames de petróleo en los mares, ríos y lagos, produciendo la contaminación ambiental y generando grades daños en la fauna marina, aves y vegetación, además esto también perjudica en gran parte a la pesca y las actividades recreativas en las diferentes playas. Y por qué no también mencionar el humo de las grandes fábricas industriales de los países productores. Para las personas que transitamos diariamente por las calles en donde vivimos es común percibir el humo negro que sale de los tubos de escape de los vehículos, buses, y motos esto también afecta al medio ambiente y sobre todo a la salud de las personas, siendo muchas las causas y efectos que nos perjudican, deberíamos tomar medidas para evitar que nuestro planeta se siga degradando.

\section{Plástico}

Según: (Alliance G. 2016., Durán E. 2014., Lord R. 2016., Laborda J. 2014., Ortiz M. 2015 \& Villena A. 2015). Aseguran que, la producción masiva de plástico se inició en la década de 1950 y ha aumentado exponencialmente, la palabra pastico también se usa para describir un grupo de componentes artificiales o de fibras sintética que derivan del gas o del petróleo, existen varios factores que influyen en la duración de un plástico, la degradación por radiación ultravioleta, mientras más luz exista más degradación sufre el plástico por los rayos ultravioleta, además de influir la orientación al sol de la lámina plástica. Los plásticos con aditivos foto estabilizadores se degradan más lentamente. Los plásticos de un solo uso son una fuente importante de basura marina artículos como: bolsas, botellas y sorbetes representan un peligro físico para la vida marina.

Imagen N. 2: Residuos de Plásticos.

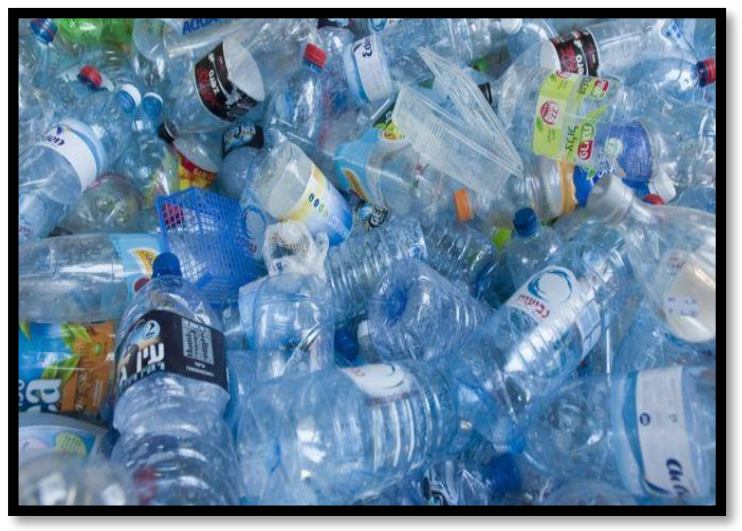

Fuente: https://www.bbc.com/mundo/noticias-40664725

El plástico es un material sintético que puede ser moldeado de diferentes formas y tamaños y todas las personas cada día utilizamos productos sin tener la menor idea de las consecuencias que pueden ocasionar tal es el caso de los plásticos como fundas, botellas, platos, vasos sorbetes etc. Esta clase de plástico se utiliza en gran cantidad debido a su 
uso tan común, por esta razón pensemos que son indefensos, por lo tanto, cuando los desechamos tardan mucho tiempo de descomponerse ya que no son reutilizables y no son biodegradables y además causa mucho daño a nuestra salud y al medio ambiente. En las islas Galápagos específicamente en la isla San Cristóbal, se está trabajando mucho para el cuidado y mantenimiento de la islas conjuntamente con las diferentes entidades públicas como es el Gad Municipal de San Cristóbal, el Parque Nacional Galápagos, Consejo de Gobierno del Régimen Especial de Galápagos y el Centro de Gestión de Residuos sólidos, realizando limpiezas costeras de superficie y submarinas a esta labor también de integran los pobladores de las islas los jóvenes estudiantes de los colegios y también con la ayuda de la Segunda Zona Naval, de esta manera se recolecta grandes cantidades de basura plástica y ayudan de esta forma al cuidado de las islas, ya que la contaminación por este tipo de residuos no solo es visual sino también de gran impacto ambiental afectando a las especies y animales que habitan en el Archipiélago, aves como pinzones y tortugas confunden los plásticos como alimentos ingiriéndolos y les causa la muerte, otros como los lobos marinos juegan con loa plásticos y se quedan atrapados o enredados. El uso de tantas fundas de plástico causa grandes problemas en el mundo por que se consume grandes cantidades de petróleo para su elaboración y cuando se desechan forman grandes cantidades de basura.

Gráfico N. 1: Datos Estadísticos de Jornada de Limpieza Costera.

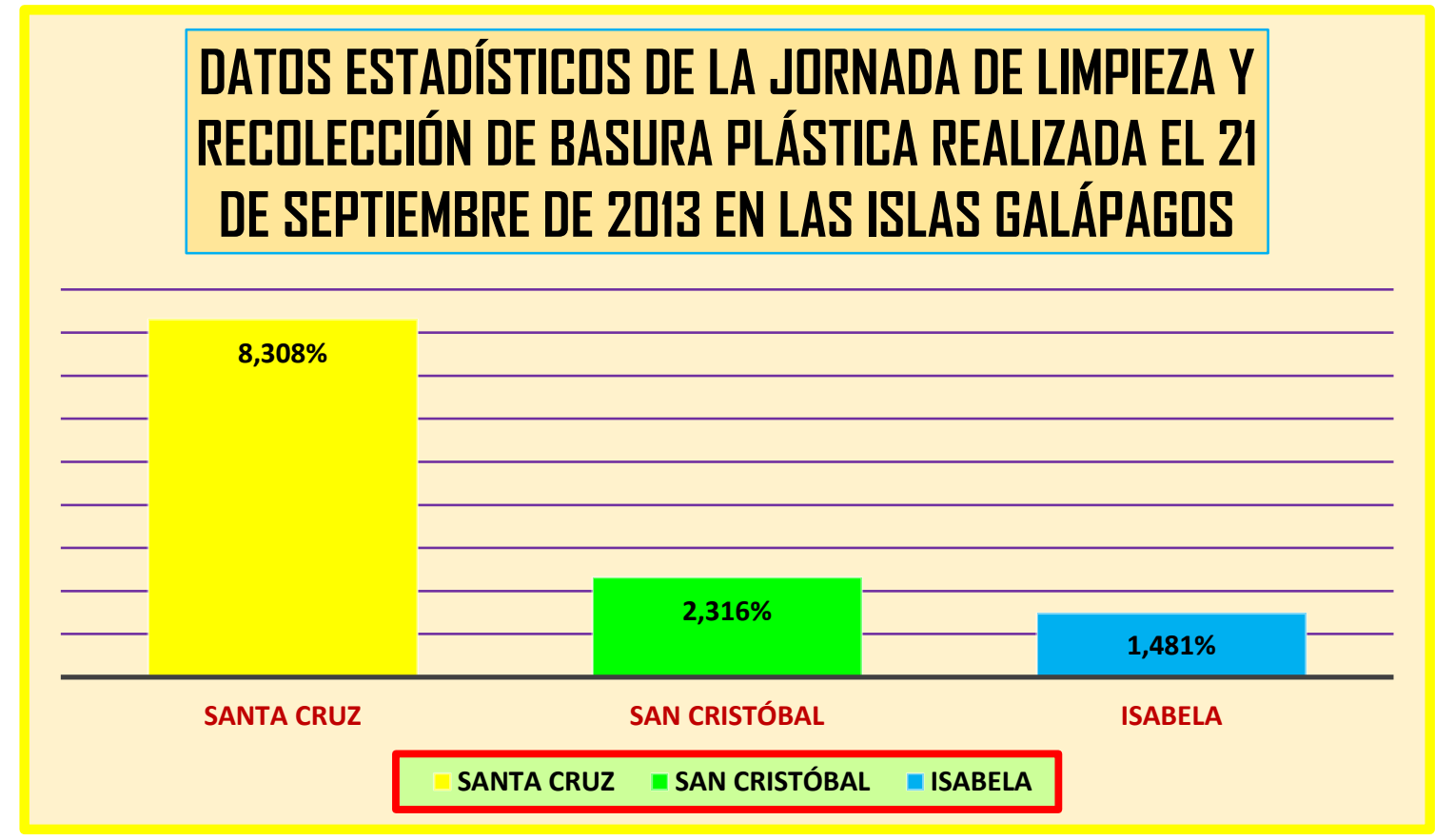

Elaborado por: Fanny Yupangui

Según la información tomada del Diario el Universo afirman, que el 24 de septiembre de 2013 se realizó en las islas Galápagos la jornada de limpieza costera, dirigido por el Parque Nacional Galápagos, en la cual en dicha actividad se reunió 750 voluntarios provenientes de la Islas Santa Cruz, San Cristóbal e Isabela conjuntamente con las diferentes entidades como el Ministerio de Ambiente, el gobierno de Santa Cruz, Gobierno de San Cristóbal, Gobierno de Isabela y la Policía Nacional, así como 
organizadores del sector pesquero, en esta actividad se pudo recolectar en Santa Cruz 8.308 libras, San Cristóbal 2.316 libras e Isabela 1.481 libras de desechos, dando así un total de 12.105 libras de desechos recolectados en las Islas Galápagos.

Gráfico N. 2: Datos Estadísticos recolección de Basura plástica hasta abril de 2018

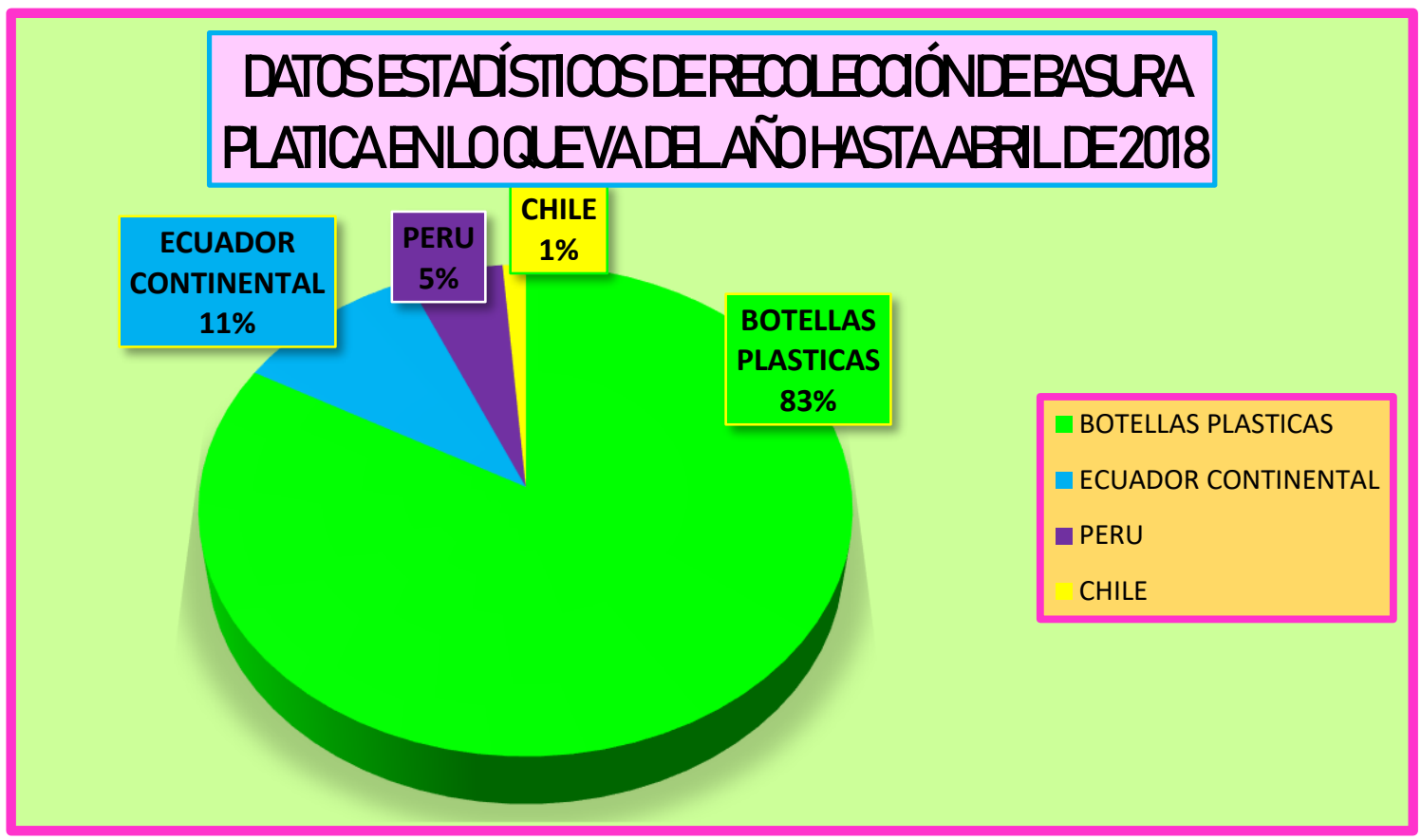

Elaborado por: Fanny Yupangui

Conforme con la información tomada del Periódico La Hora, señalan que en el transcurso del año hasta abril de 2018 se han recolectado 22 toneladas de basura siendo el $80 \%$ botellas plásticas, también la mayoría de estos desechos provienen de Chile y Perú, atraídas por las corrientes marinas llegando así a las Islas Galápagos, como dato importante tenemos que el plástico tarda entre 100 a 1.000 años en descomponerse, y una botella de plástico tarda una media de 500 años en desintegrarse.

Imagen N. 3: Basura marina mata a los animales de las Galápagos

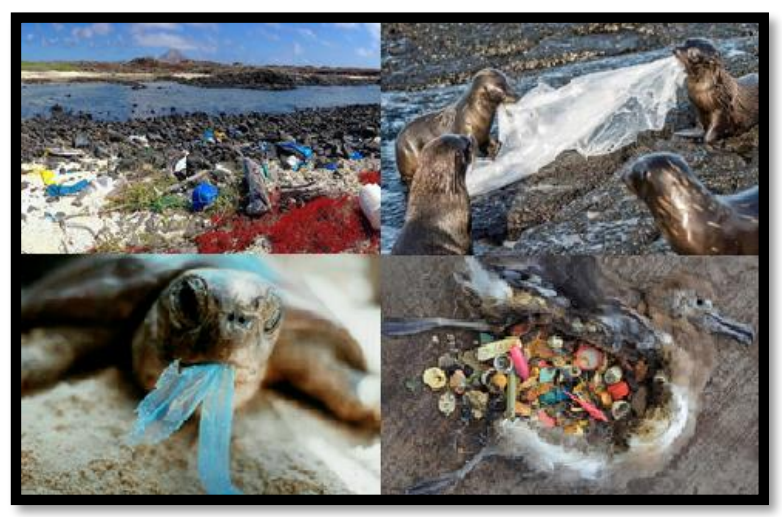

Fuente: https://fundacionmundosinfronteras.org/atraccion-fatal-tortugas-yplastico 


\section{Galápagos}

Según: (Carrión J. 2014., Cruz M. 2015., CRUZ E. 2015., Grosberg M. 2016., Guides R. 2016 \& Nicholls H. 2014). Aseguran que, las islas Galápagos son uno de los lugares más increíbles del planeta con ecosistemas y especies únicas tanto en su parte terrestre como marina. Las islas se formaron hace 3 a 5 millones de años, cuando volcanes submarinos entraron en erupción produciendo una biodiversidad singular y frágil. Estas características hacen que las islas sean reconocidas como Patrimonio Natural de la Humanidad y que tanto el estado Ecuatoriano como Organismos Internacionales lo protejan bajo diferentes figuras: Parque Nacional, Reserva Marina, Reserva de la Biosfera. Esto hace que sus habitantes sean comprometidos con la conservación de la biodiversidad y ecosistemas de su patrimonio natural. En el territorio Galapaguense es la provincia menos poblada del país debido principalmente al afán de conservar al máximo la flora y fauna de la región, es el mayor centro turístico del Ecuador, así como también una de las reservas ecológicas más grandes e imponentes del planeta, las actividades principales de la provincia son el turismo debido a su calidad de reserva natural, y la pesca.

Imagen N. 4: Límite geográfico de las Islas Galápagos.

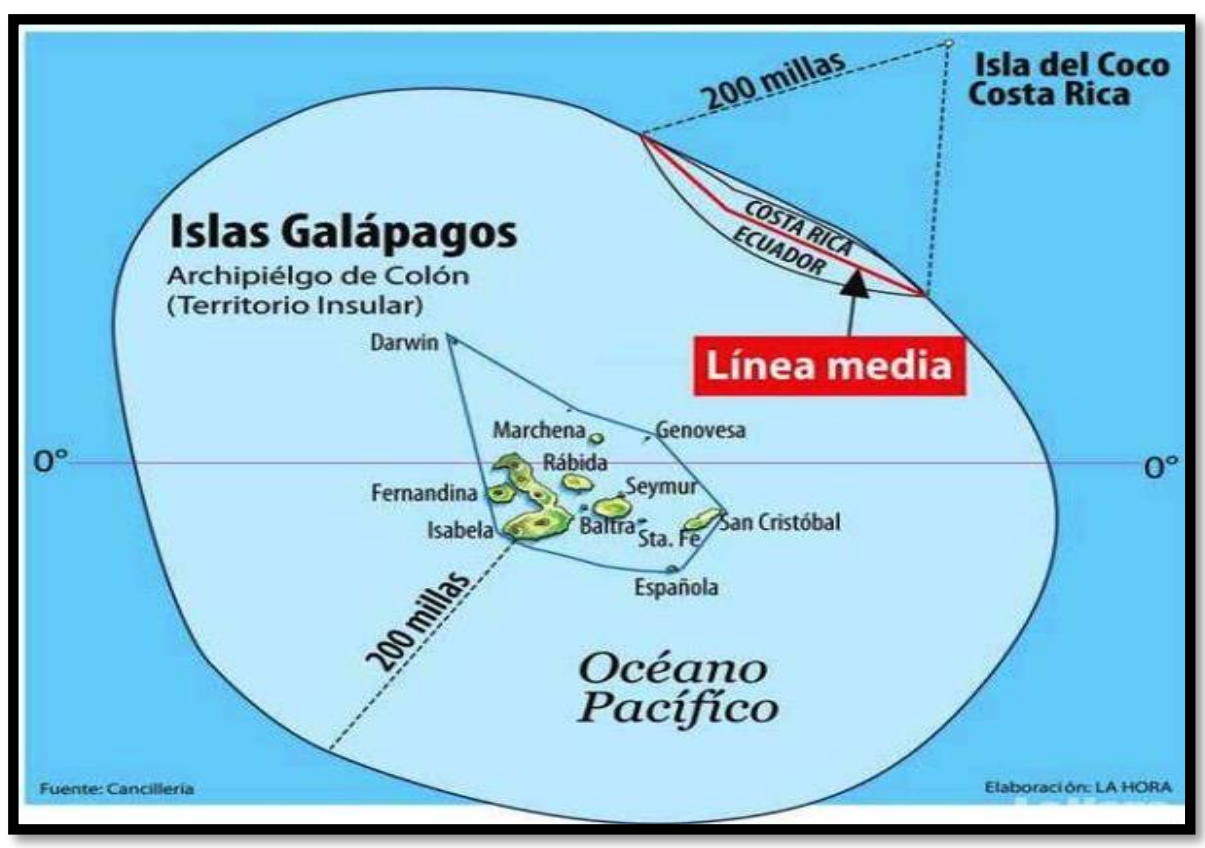

Fuente: https://www.elpais.cr/2016/09/08/limites-marinos-con-ecuador-unacronica/

Las islas Galápagos conocidas también como las islas encantadas, es el habitad de muchas especies endémicas, con flora y fauna únicas en el mundo, un lugar maravilloso en la que los residentes de la islas junto con las organizaciones como el Parque Nacional y la Reserva Marina, tiene la obligación de protegerlas y conservarlas para que ese pedazo de paraíso no desaparezca. Las Galápagos son visitadas por todos los turistas nacionales y extranjeros de todo el mundo dando así su mayor fuente de ingreso al turismo y la pesca, 
también se desarrolló el turismo ecológico con el fin de preservar las especies, estas islas fueron el habitad del solitario George la última especie de las tortugas gigantes de la Isla Pinta, se estima que la formación de la primera isla tuvo lugar hace 5 millones de años como resultado de una actividad tectónica, las islas en donde todavía existen volcanes activos en proceso de erupción es el las islas Fernandina e Isabela. Actualmente Galápagos es la Provincia de Ecuador y está constituida por 3 cantones que son: San Cristóbal, Santa Cruz e Isabela.

Imagen N. 5: Islas Galápagos.

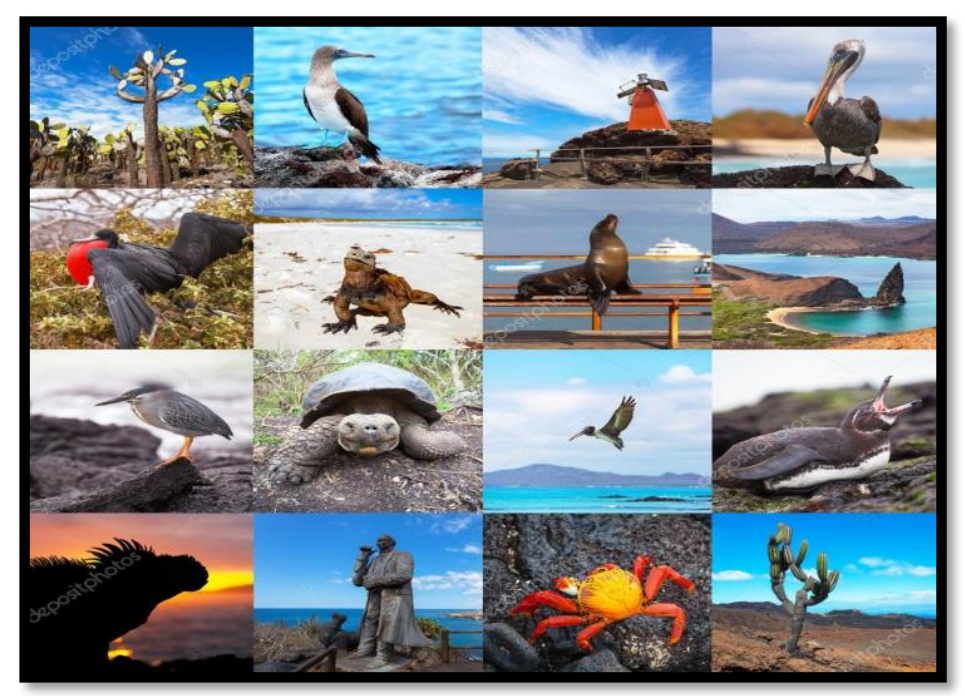

Fuente: https://sp.depositphotos.com/75206657/stock-photo-set-of-famousplaces-and.html

\section{Conclusiones}

$>$ El impacto ambiental de basura plástica no solo afecta a las islas Galápagos y a sus especies, sino también a todo el territorio costanero del Ecuador y del mundo, esto generado por el hombre y su irresponsabilidad hacia el medio en el vivimos.

$>$ La basura plástica genera una alta contaminación ya que está compuesta por materiales como la fibra sintética derivada del petróleo y esto hace que tome mucho tiempo para su desintegración afectando así en ese transcurso de tiempo a las especies marinas.

$>$ Las islas encantadas o las islas Galápagos como se las conoce, es el habitad de muchas especies únicas en el mundo tanto las terrestres como marinas, este singular archipiélago está sufriendo un cambio por la contaminación de basura plástica ya que esta basura llega al mar y se convierte en un peligro para las especies que allí habitan generando así la muerte de las mismas.

$>$ Es responsabilidad de todas las personas el cuidado no solo de las Galápagos sino también de todo el Planeta Tierra ya que no solo perjudicamos a las plantas y 
animales si no también nos perjudicamos nosotros mismos y a nuestras generaciones. Si queremos ayudar a que esto no siga sucediendo no botemos basura en las calles, reciclemos, reutilicemos, así dejaremos un mejor Planeta a nuestras generaciones.

\section{Referencias Bibliográficas}

Alliance, G. (10 de 2016). SINGLE USE PLASTICS. Obtenido de https://ieep.eu/archive_uploads/2128/IEEP_ACES_Product_Fiche_Single_Use _Plastics_Final_October_2016.pdf

Allinson, R. (12 de 03 de 2014). Environmental impacts. Obtenido de https://ec.europa.eu/research/evaluations/pdf/archive/other_reports_studies_an d_documents/envti0413167enn_002.pdf

CALlEJA, D. (2018). Motores 2. a edición 2018. Esoaña: Ediciones Paraninfo, S.A.

Carrión, J. (15 de 02 de 2014). www.galapagos.org. Obtenido de https://www.galapagos.org/wpcontent/uploads/2015/08/InformeGalapagos_2013-2014-5-Carrion-Tacuriarticle.pdf

CRUZ, E. (12 de 02 de 2015). www.gobiernogalapagos.gob.ec. Obtenido de http://www.gobiernogalapagos.gob.ec/wpcontent/uploads/downloads/2015/09/propuesta-plan-galapagos-version14_sep_2015.pdf

Cruz, M. (2 de 01 de 2015). bioseguridadgalapagos.gob.ec. Obtenido de http://bioseguridadgalapagos.gob.ec/wpcontent/uploads/downloads/imgcom2018/planestrategicoinstitucionalabg.pdf

Durán, E. (2014). Operaciones culturales, recolección, almacenamiento y envasado de productos. AGAX0208. Bolivia: IC Editorial, 2014.

FERNÁNDEZ，J. (2014). Equipos eléctricos y electrónicos 2. a edición 2018. España: Ediciones Paraninfo, S.A., 2014.

Grosberg, M. (2016). Ecuador y las islas Galápagos 6. España: Grupo Planeta Spain, 2016.

Guides, R. ( 2016). The Rough Guide to Ecuador \& the Galápagos Islands. España: Rough Guides UK, 2016.

Iván, V. (2016). Derecho e innovación ambiental: Enseñanzas de un caso colombiano. Bogota: Editorial Universidad del Rosario, 2016.

Laborda, J. (2014). Quilo de Ciencia Volumen VI. Mexico: Lulu.com, 2014. 
Lord, R. (12 de 07 de 2016). plastics.americanchemistry.com. Obtenido de https://plastics.americanchemistry.com/Plastics-and-Sustainability.pdf

Loustaunau, M. (21 de 05 de 2014). Aspectos e Impactos Ambientales . Obtenido de https://www.fing.edu.uy/iq/cursos/proyectoindustrial/A\&IA.pdf

Nicholls, H. ( 2014). The Galapagos. Canada: Profile Books, 2014.

Ortiz, M. (2015). Tendencias de innovación en la ingeniería de alimentos. España: OmniaScience, 2015.

Padova, U. (9 de 06 de 2014). Environmental Impact Assessment. Obtenido de http://lasa.dii.unipd.it/via/LEX/2_via.pdf

Villena, A. (5 de 07 de 2015). www.ecologistasenaccion.org. Obtenido de https://www.ecologistasenaccion.org/IMG/pdf/informe-plastico.pdf 
Para citar el artículo indexado.

Carrasco T. (2018). La utilización de plásticos y su impacto ambiental en las Islas Galápagos. Revista electrónica Explorador Digital 2(2), 16-25. Recuperado desde: http://cienciadigital.org/revistacienciadigital2/index.php/exploradordigital/article/view/329/7 $\underline{41}$

\section{Ciencia Digital \\ Editorial}

El artículo que se publica es de exclusiva responsabilidad de los autores y no necesariamente reflejan el pensamiento de la Revista Explorador Digital.

El articulo queda en propiedad de la revista y, por tanto, su publicación parcial y/o total en otro medio tiene que ser autorizado por el director o editor de la Revista Explorador Digital.
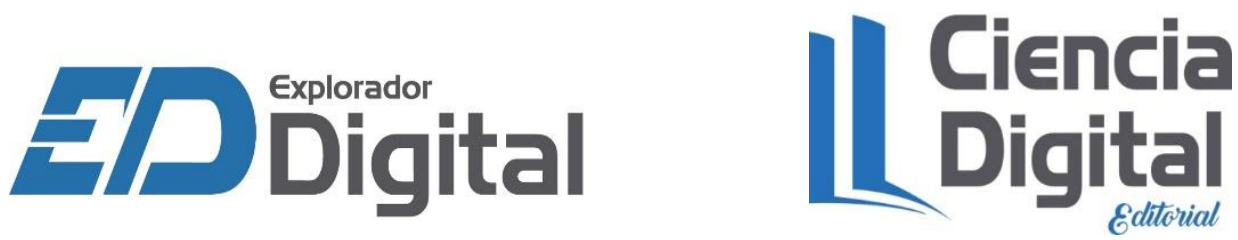